University of New Mexico

UNM Digital Repository

Mathematics and Statistics Faculty and Staff

Publications

Academic Department Resources

2020

A New Trend to Extensions of Cl-algebras

Florentin Smarandache

Akbar Rezaei

Hee Sik Kim

Follow this and additional works at: https://digitalrepository.unm.edu/math_fsp

Part of the Algebra Commons, and the Algebraic Geometry Commons 


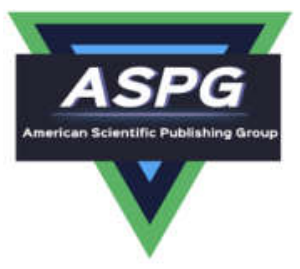

\title{
A New Trend to Extensions of CI-algebras
}

\author{
${ }^{1}$ Florentin Smarandache, ${ }^{2, *}$ Akbar Rezaei, ${ }^{3}$ Hee Sik Kim \\ ${ }^{1}$ Department of Mathematics \& Sciences, University of New Mexico, Gallup, NM 87301, USA. \\ E-mail: smarand@unm.edu \\ ${ }^{2}$ Department of Mathematics, Payame Noor University, P.O. Box. 19395-3697, Tehran, Iran. \\ E-mail: rezaei@pnu.ac.ir \\ ${ }^{3}$ Department of Mathematics, Hanyang University, Seoul 04763, Korea. \\ E-mail: heekim@hanyang.ac.kr \\ * Correspondence: rezaei@pnu.ac.ir
}

\begin{abstract}
In this paper, as an extension of CI-algebras, we discuss the new notions of Neutro-CI-algebras and Anti-CI-algebras. First, some examples are given to show that these definitions are different. We prove that any proper CI-algebra is a Neutro-BE-algebra or Anti-BE-algebra. Also, we show that any NeutroSelf-distributive and AntiCommutative CIalgebras are not BE-algebras.
\end{abstract}

Keywords: CI-algebra, Neutro-CI-algebra, Anti-CI-algebra, Self-distributive, NeutroSelf-distributive, AntiSelfdistributive, Commutative, NeutroCommuative, AntiCommutative.

\section{Introduction}

H.S. Kim et al. introduced the notion of BE-algebras as a generalization of dual BCK-algebras [1]. A. Walendziak defined the notion of commutative BE-algebras and discussed some of their properties [11]. A. Rezaei et al. investigated the relationship between Hilbert algebras and BE-algebras [5]. B.L. Meng introduced the notion of CIalgebras as a generalization of BE-algebras and dual BCI/BCK-algebras, and studied some relations with BE-algebras [2]. Then he defined the notion of atoms in CI-algebras and singular CI-algebras and investigated their properties [3]. Filters and upper sets were studied in detail by B. Piekart et al. [4].

Recently, in 2019-2020 F. Smarandache [8, 9, 10] constructed for the first time the neutrosophic triple corresponding to the Algebraic Structures as (Algebraic Structure, NeutroAlgebraic Structure, AntiAlgebraic Structure), where a (classical) Algebraic Structure is an algebraic structure dealing only with (classical) Operations) (that are totally well-defined) and (classical) Axioms (that are totally true). A NeutroAlgebraic Structure is an algebraic structure that has at least one NeutroOperation or NeutroAxiom, and no AntiOperation and no AntiAxiom, while an AntiAlgebraic Structure is an algebraic structure that has at least one AntiOperation or one AntiAxiom. Moreover, some left (right)-quasi neutrosophic triplet structures in BE-algebras were studied by X. Zhang et al. [12]. 
The aim of this paper is to characterize these definitions to CI-algebras. Also, the notions of NeutroSelfdistributive / AntiSelf-distributive and NeutroCommutative / AntiCommutative in CI-algebras are studied. Finally, as an alternative to the definition of CI-algebra, Neutro-CI-algebra and Anti-CI-algebra are defined.

\section{Preliminaries}

In this section we recall some basic notions and results regarding CI-algebras and BE-algebras. CI-algebras were introduced in [2] as a generalization of BE-algebras (see [1]) and properties of them have recently been studied in [3] and [4].

Definition 2.1. ([2]) A CI-algebra is an algebra $(X, \rightarrow, 1)$ of type $(2,0)$ (i.e. $X$ is a non-empty set, $\rightarrow$ is a binary operation and 1 is a constant element) satisfying the following axioms, for all $x, y, z \in X$ :

(CI1) $(\forall x \in X)(x \rightarrow x=1)$

$(\mathrm{CI} 2)(\forall x \in X)(1 \rightarrow x=x)$;

(CI3) $(\forall x, y, z \in X$, with $x \neq y)(x \rightarrow(y \rightarrow z)=y \rightarrow(x \rightarrow z))$.

We introduce a binary relation $\leq$ on $X$ by $x \leq y$ if and only if $x \rightarrow y=1$. A CI-algebra $(X, \rightarrow, 1)$ is said to be a BE-algebra ([1]) if

(BE) $(\forall x \in X)(x \rightarrow 1=1)$.

By $(\mathrm{CI} 1) \leq$ is only reflexive.

In what follows, let $X$ be a CI-algebra unless otherwise specified. A CI-algebra $X$ is proper if it is not a BE-algebra.

For example, the set $X=\{1, a\}$, with the following Cayley table is a proper CI-algebra, since $a \rightarrow 1=a \neq 1$.

Table 1

\begin{tabular}{|l|l|l|}
\hline$\rightarrow$ & 1 & $\mathrm{a}$ \\
\hline 1 & 1 & $\mathrm{a}$ \\
\hline $\mathrm{a}$ & $\mathrm{a}$ & 1 \\
\hline
\end{tabular}

Theorem 2.2. Let $(X, \rightarrow, 1)$ be a CI-algebra. The binary operation $\rightarrow$ is associative if and only if $x \rightarrow 1=x$, for all $x \in X$.

Proof. Assume that $\rightarrow$ is associative. Using (CI2) and associativity, we have

$$
x=1 \rightarrow x=(x \rightarrow x) \rightarrow x=x \rightarrow(x \rightarrow x)=x \rightarrow 1 .
$$

Conversely, suppose that $x \rightarrow 1=x$, for all $x \in X$. Let $x, y, z \in X$, then by applying assumption and three times (CI3), we get

$(x \rightarrow y) \rightarrow z=(x \rightarrow y) \rightarrow(z \rightarrow 1)=z \rightarrow((x \rightarrow y) \rightarrow 1)=z \rightarrow(x \rightarrow y)=z \rightarrow(x \rightarrow(y \rightarrow 1))=x \rightarrow(\rightarrow(y \rightarrow$ 1)) $=x \rightarrow(y \rightarrow(z \rightarrow 1))=x \rightarrow(y \rightarrow z)$.

Thus, $(x \rightarrow y) \rightarrow z=x \rightarrow(y \rightarrow z)$.

Also, if $\rightarrow$ is associative relation, then CI-algebra $(X, \rightarrow, 1)$ is an Abelian group with identity 1 , since 


$$
x \rightarrow y=x \rightarrow(y \rightarrow 1)=y \rightarrow(x \rightarrow 1)=y \rightarrow x .
$$

Example 2.3. (i) Let $\mathbb{R}$ be the set of all real numbers and $\rightarrow$ be the binary operation on $\mathbb{R}$ defined by $x \rightarrow y=y \div x$, where $\div$ is the binary operation of division. Then $(\mathbb{R}-\{0\}, \rightarrow, 1)$ is a CI-algebra, but it is not a BE-algebra.

(CI1) holds, since for every $0 \neq x \in \mathbb{R}, x \rightarrow x=x \div x=1$;

(CI2) valid, since for all $x \in X, 1 \rightarrow x=x$;

(CI3) holds. Let $x, y, z \in X$. Then we have

$x \rightarrow(y \rightarrow z)=x \rightarrow(z \div y)=(z \div y) \div x=(z \div x) \div y=y \rightarrow(z \div x)=y \rightarrow(x \rightarrow z)$.

(BE) is not valid, since $5 \rightarrow 1=1 \div 5=\frac{1}{5} \neq 1$. Thus, $(\mathbb{R}-\{0\}, \rightarrow, 1)$ is a proper CI-algebra.

(ii) Consider the real interval $[0,1]$ and let $\rightarrow$ be the binary operation on $[0,1]$ defined by $x \rightarrow y=1-x+x y$. Then $([0,1], \rightarrow, 1)$ is not a CI-algebra (so, is not a BE-algebra), since (CI1) and (CI3) are not valid. Note that (BE) holds, since $x \rightarrow 1=1-x+x \cdot 1=1-x+x=1$.

Proposition 2.4. ([2]) Let $\boldsymbol{X}$ be a CI-algebra. Then for all $\boldsymbol{x}, \boldsymbol{y} \in \boldsymbol{X}$,

(i) $\quad y \rightarrow((y \rightarrow x) \rightarrow x)=1$;

(ii) $\quad(x \rightarrow \mathbf{1}) \rightarrow(y \rightarrow \mathbf{1})=(x \rightarrow y) \rightarrow \mathbf{1}$.

Definition 2.5. ([1, 2]) A CI/BE-algebra $\boldsymbol{X}$ is said to be self-distributive if for any $\boldsymbol{x}, \boldsymbol{y}, \mathbf{z} \in \boldsymbol{X}$,

$$
x \rightarrow(y \rightarrow z)=(x \rightarrow y) \rightarrow(x \rightarrow z) .
$$

Example 2.6. Consider the CI-algebra given in Example 2.3 (i). It is not self-distributive. Let $\boldsymbol{x}:=\mathbf{5}, \boldsymbol{y}:=\mathbf{4}$ and $z:=7$. Then we have $5 \rightarrow(4 \rightarrow 7)=5 \rightarrow \frac{7}{4}=\frac{7}{20} \neq(5 \rightarrow 4) \rightarrow(5 \rightarrow 7)=\frac{4}{5} \rightarrow \frac{7}{5}=\frac{7}{4}$.

Proposition 2.7. ([2]) Every self-distributive CI-algebra $\boldsymbol{X}$ is a BE-algebra.

Note that if $\boldsymbol{X}$ is self-distributive, then $\leq$ is transitive ([6]).

Definition 2.8. ([2, 5, 11]) A CI/BE-algebra X is said to be commutative if for any $\boldsymbol{x}, \boldsymbol{y} \in \boldsymbol{X}$,

$$
x \rightarrow(x \rightarrow y)=y \rightarrow(y \rightarrow x) .
$$

Example 2.9. ([6]) (i) Let $\mathbb{N}$ be the set of all natural numbers and $\rightarrow$ be the binary operation on $\mathbb{N}$ defined by

$$
x \rightarrow y= \begin{cases}y & \text { if } x=1 \\ 1 & \text { otherwise. }\end{cases}
$$

Then $(\mathbb{N}, \rightarrow, \mathbf{1})$ is a non-commutative BE-algebra.

(ii) Let $\mathbb{N}_{\mathbf{0}}=\mathbb{N} U\{\boldsymbol{0}\}$ and let $\rightarrow$ be the binary operation on $\mathbb{N}_{\mathbf{0}}$ defined by

$$
x \rightarrow y= \begin{cases}0 & \text { if } x \geq y \\ y-x & \text { otherwise }\end{cases}
$$

10.5281/zenodo.3788124 
Then $\left(\mathbb{N}_{\mathbf{0}}, \rightarrow, \mathbf{0}\right)$ is a commutative BE-algebra ([6]), but it is not self-distributive, since

$$
5 \rightarrow(6 \rightarrow 7)=5 \rightarrow 1=0 \neq(5 \rightarrow 6) \rightarrow(5 \rightarrow 7)=1 \rightarrow 2=1 .
$$

Proposition 2.10. ([2]) Every commutative CI-algebra $X$ is a BE-algebra.

Note that if $\boldsymbol{X}$ is commutative, then $\leq$ is anti-symmetric ([6]). Hence, if $\boldsymbol{X}$ is a commutative and self-distributive CIalgebra, then $\leq$ is a partially ordered set $([6])$.

\section{On NeutroSelf-distributive and AntiSelf-distributive CI-algebras}

Definition 3.1. A CI-algebra $\boldsymbol{X}$ is said to be NeutroSelf-distributive if

$$
(\exists x, y, z \in X)(x \rightarrow(y \rightarrow z)=(x \rightarrow y) \rightarrow(x \rightarrow z)) \text { and }(\exists x, y, z \in X)(x \rightarrow(y \rightarrow z) \neq(x \rightarrow y) \rightarrow(x \rightarrow z))
$$

Example 3.2. Consider the non-self-distributive CI-algebra given in Example 2.3 (i). If $\boldsymbol{x}:=\mathbf{1}$, then for all $\boldsymbol{y}, \boldsymbol{z} \in \mathbb{R}-$ $\{\mathbf{0}\}$, we have $\boldsymbol{x} \rightarrow(\boldsymbol{y} \rightarrow \boldsymbol{z})=(\boldsymbol{x} \rightarrow \boldsymbol{y}) \rightarrow(\boldsymbol{x} \rightarrow \boldsymbol{z})$. If $\boldsymbol{x} \neq \mathbf{1}$, then for all $\boldsymbol{y}, \boldsymbol{z} \in \mathbb{R}-\{\mathbf{0}\}$, we have $\boldsymbol{x} \rightarrow(\boldsymbol{y} \rightarrow \boldsymbol{z}) \neq$ $(\boldsymbol{x} \rightarrow \boldsymbol{y}) \rightarrow(\boldsymbol{x} \rightarrow \boldsymbol{z})$. Hence $(\mathbb{R}-\{\mathbf{0}\}, \rightarrow, \mathbf{1})$ is a NeutroSelf-distributive CI-algebra.

Definition 3.3. A CI-algebra $\boldsymbol{X}$ is said to be AntiSelf-distributive if

$$
(\forall x, y, z \in X, \text { with } x \neq 1)(x \rightarrow(y \rightarrow z) \neq(x \rightarrow y) \rightarrow(x \rightarrow z)) .
$$

Example 3.4. Consider the CI-algebra given in Example 2.3 (i). Then it is an AntiSelf-distributive CI-algebra, since for all $\boldsymbol{x}, \boldsymbol{y}, \boldsymbol{z} \in \mathbb{R}-\{\boldsymbol{0}\}$ and $\boldsymbol{x} \neq \mathbf{1}$, we can see that

$$
x \rightarrow(y \rightarrow z)=(z \div y) \div x=\frac{z}{y x} \neq(x \rightarrow y) \rightarrow(x \rightarrow z)=(z \div x) \div(y \div x)=\frac{z}{y} .
$$

Theorem 3.5. Let $\boldsymbol{X}$ be an AntiSelf-distributive CI-algebra. Then $\boldsymbol{X}$ is not a BE-algebra.

Proof. Assume that $\boldsymbol{X}$ is an AntiSelf-distributive CI-algebra and $\mathbf{1} \neq \boldsymbol{x} \in \boldsymbol{X}$. Take $\boldsymbol{y}=\boldsymbol{z}=\mathbf{1}$ and using AntiSelfdistributivity and applying (CI1) two times, we have

$x \rightarrow 1=x \rightarrow(1 \rightarrow 1) \neq(x \rightarrow 1) \rightarrow(x \rightarrow 1)=1$.

Thus, $(\forall x \in X$, with $x \neq \mathbf{1})(\boldsymbol{x} \rightarrow \mathbf{1} \neq \mathbf{1})$, and so $\boldsymbol{X}$ is not a BE-algebra.

Corollary 3.6. There is no AntiSelf-distributive BE-algebra.

Proposition 3.7. Let $\boldsymbol{X}$ be an AntiSelf-distributive CI-algebra. Then

$$
(\forall x, y \in X, \text { with } x \neq 1)(x \rightarrow(x \rightarrow y) \neq x \rightarrow y)
$$

Proof. Let $\boldsymbol{X}$ be a CI-algebra and $\boldsymbol{x}, \boldsymbol{y} \in \boldsymbol{X}$. Using AntiSelf-distributivity and (CI2), we get

$$
x \rightarrow(x \rightarrow y) \neq(x \rightarrow x) \rightarrow(x \rightarrow y)=1 \rightarrow(x \rightarrow y)=x \rightarrow y .
$$

Thus, $x \rightarrow(x \rightarrow y) \neq x \rightarrow y$. 
Proposition 3.8. Let $\boldsymbol{X}$ be an AntiSelf-distributive CI-algebra, and $\boldsymbol{x} \leq \boldsymbol{y}$. Then $\boldsymbol{z} \rightarrow \boldsymbol{x} \leq \boldsymbol{z} \rightarrow \boldsymbol{y}$, for all $\mathbf{1} \neq \boldsymbol{z} \in \boldsymbol{X}$.

Proof. Suppose that $\boldsymbol{X}$ is an AntiSelf-distributive CI-algebra, $\boldsymbol{x} \leq \boldsymbol{y}$ and $\mathbf{1} \neq \boldsymbol{z} \in \boldsymbol{X}$. Then $\boldsymbol{x} \rightarrow \boldsymbol{y}=\mathbf{1}$. Applying AntiSelf-distributivity and (BE), we get

$$
(z \rightarrow x) \rightarrow(z \rightarrow y) \neq z \rightarrow(x \rightarrow y)=z \rightarrow 1 \neq 1
$$

Thus, $z \rightarrow x \notin z \rightarrow y$, for all $1 \neq z \in X$.

Proposition 3.9. Let $\boldsymbol{X}$ be an AntiSelf-distributive CI-algebra. Then $\leq$ is not transitive.

Proof. Suppose that $\boldsymbol{X}$ is an AntiSelf-distributive CI-algebra, $\boldsymbol{x} \leq \boldsymbol{y}$ and $\boldsymbol{y} \leq \boldsymbol{z}$. Then $\boldsymbol{x} \rightarrow \boldsymbol{y}=\mathbf{1}$ and $\boldsymbol{y} \rightarrow \boldsymbol{z}=\mathbf{1}$. Using (CI2) and AntiSelf-distributivity, we have

$$
x \rightarrow z=1 \rightarrow(x \rightarrow z)=(x \rightarrow y) \rightarrow(x \rightarrow z) \neq x \rightarrow(y \rightarrow z)=x \rightarrow 1 \neq 1
$$

Thus, $\boldsymbol{x} \not \mathbf{z}$.

\section{On NeutroCommutative and AntiCommutative CI-algebras}

Definition 4.1. A CI/BE-algebra $\boldsymbol{X}$ is said to be NeutroCommutative if

$$
(\exists x, y \in X \text { with } x \neq y)(x \rightarrow(x \rightarrow y)=y \rightarrow(y \rightarrow x)) \text { and }(\exists x, y \in X)(x \rightarrow(x \rightarrow y) \neq y \rightarrow(y \rightarrow x))
$$

Example 4.2. (i) Consider the non-commutative BE-algebra given in Example 2.9 (i). If $\boldsymbol{x}, \boldsymbol{y} \in \mathbb{N}-\{\mathbf{1}\}$, then $\boldsymbol{x} \rightarrow$ $(x \rightarrow y)=y \rightarrow(y \rightarrow x)$. If $x=1$ and $y \neq 1$, then $x \rightarrow(x \rightarrow y)=y \neq y \rightarrow(y \rightarrow x)=1$.

(ii) Consider the CI-algebra given in Example 2.3 (i). Then it is not a NeutroCommutative CI-algebra, since, for all $\boldsymbol{x}, \boldsymbol{y} \in \mathbb{R}-\{\boldsymbol{0}\}$, we have $\boldsymbol{x} \rightarrow(\boldsymbol{x} \rightarrow \boldsymbol{y}) \neq \boldsymbol{y} \rightarrow(\boldsymbol{y} \rightarrow \boldsymbol{x})$, only if $\boldsymbol{x}=\boldsymbol{y}=\mathbf{1}$, then $\boldsymbol{x} \rightarrow(\boldsymbol{x} \rightarrow \boldsymbol{y})=\boldsymbol{y} \rightarrow(\boldsymbol{y} \rightarrow \boldsymbol{x})$. Thus, there is not $\boldsymbol{x} \neq \boldsymbol{y}$ such that $\boldsymbol{x} \rightarrow(\boldsymbol{x} \rightarrow \boldsymbol{y})=\boldsymbol{y} \rightarrow(\boldsymbol{y} \rightarrow \boldsymbol{x})$.

Definition 4.3. A CI/BE-algebra $\boldsymbol{X}$ is said to be AntiCommutative if

$$
(\forall x, y \in X \text { with } x \neq y)((x \rightarrow y) \rightarrow y \neq(y \rightarrow x) \rightarrow x)
$$

Example 4.4. Consider the CI-algebra given in Example 2.3 (i). Then it is an AntiCommutative CI-algebra.

Proposition 4.5. Let $\boldsymbol{X}$ be an AntiCommutative CI-algebra. Then $\boldsymbol{X}$ is not a BE-algebra.

Proof. By contrary, let $\boldsymbol{X}$ be a BE-algebra. Then for all $\boldsymbol{x} \in \boldsymbol{X}, \boldsymbol{x} \rightarrow \mathbf{1}=\mathbf{1}$. Hence $(\boldsymbol{x} \rightarrow \mathbf{1}) \rightarrow \mathbf{1}=\mathbf{1} \rightarrow \mathbf{1}=\mathbf{1}$, by assumption and (CI1). Now, applying AntiCommutaitivity and (CI2) we get

$$
1=(x \rightarrow 1) \rightarrow 1 \neq(1 \rightarrow x) \rightarrow x=x \rightarrow x=1 .
$$

Thus, $\mathbf{1} \neq \mathbf{1}$ which is a contradiction.

Corollary 4.6. There is no AntiCommutative BE-algebra.

Proposition 4.7. Let $\boldsymbol{X}$ be an AntiCommutative CI-algebra. If $\boldsymbol{x} \leq \boldsymbol{y}$, then $\boldsymbol{y} \neq(\boldsymbol{y} \rightarrow \boldsymbol{x}) \rightarrow \boldsymbol{x}$. 
Proof. Assume that $\boldsymbol{X}$ be an AntiCommutative CI-algebra and $\boldsymbol{x} \leq \boldsymbol{y}$. Then $\boldsymbol{x} \rightarrow \boldsymbol{y}=\mathbf{1}$. Using (CI2) and AntiCommutaitivity, we have

$$
y=1 \rightarrow y=(x \rightarrow y) \rightarrow y \neq(y \rightarrow x) \rightarrow x
$$

Proposition 4.8. Let $\boldsymbol{X}$ be an AntiCommutative CI-algebra. Then $\leq$ is not an anti-symmetric relation on $\boldsymbol{X}$.

Proof. Assume that $\boldsymbol{X}$ be an AntiCommutative CI-algebra. Let $\boldsymbol{x} \leq \boldsymbol{y}$ and $\boldsymbol{y} \leq \boldsymbol{x}$. Then $\boldsymbol{x} \rightarrow \boldsymbol{y}=\mathbf{1}$ and $\boldsymbol{y} \rightarrow \boldsymbol{x}=\mathbf{1}$. Applying (CI2) and AntiCommutativity, we get

$$
x=1 \rightarrow x=(y \rightarrow x) \rightarrow x \neq(x \rightarrow y) \rightarrow y=1 \rightarrow y=y .
$$

Corollary 4.9. If $\boldsymbol{X}$ is an AntiSelf-distributive or an AntiCommutative CI-algebra, then X endowed with the induced relation $\leq$ is not a partially ordered set.

Proof. By Propositions 3.9 and 4.8, we get the desired result.

If $\boldsymbol{X}$ is not a partially ordered set, then $\boldsymbol{X}$ is either totally ordered set, or totally unordered set (i.e. for any two distinct elements $\boldsymbol{x}, \boldsymbol{y} \in \boldsymbol{X}$, neither $\boldsymbol{x} \leq \boldsymbol{y}$ nor $\boldsymbol{y} \leq \boldsymbol{x}$ ).

We have the neutrosophic triplet for the order relationship $\leq$ in a similar way as for CI-algebras:

(totally ordered, partially ordered and partially unordered, totally unordered) or (Ordered, NeutroOrdered, AntiOrdered).

Corollary 4.10. If $\boldsymbol{X}$ is an AntiSelf-distributive or an AntiCommutative CI-algebra, then $\boldsymbol{x} \rightarrow(\boldsymbol{y} \rightarrow \boldsymbol{x}) \neq \mathbf{1}$, for all $x, y \in X$.

Proof. Using Corollaries 3.6 and 4.5, $\boldsymbol{X}$ is not a BE-algebra, and so applying (CI3) and (CI1) we get, for all $\boldsymbol{x}, \boldsymbol{y} \in \boldsymbol{X}$

$$
x \rightarrow(y \rightarrow x)=y \rightarrow(x \rightarrow x)=y \rightarrow 1 \neq 1
$$

\section{On Neutro-CI-algebras and Anti-CI-algebras}

The Neutro-BE-algebra and the Anti-BE-algebra as an alternative of a BE-algebra was defined in 2020 by A. Rezaei and F. Smarandache. Now, we can define Neutro-CI-algebra and Anti-CI-algebra (for detail see [7]).

\section{Definition 5.1. (Neutro-sophications)}

The Neutro-sophication of the Law (degree of well-defined, degree of indeterminacy, degree of outer-defined)

(NL) $(\exists x, y \in X)(x \rightarrow y \in X)$ and $(\exists x, y \in X)(x \rightarrow y=$ indeterminate or $x \rightarrow y \notin X)$.

The Neutro-sophication of the Axioms (degree of truth, degree of indeterminacy, degree of falsehood)

(NCI1) $(\exists x \in X)(x \rightarrow x=1)$ and $(\exists x \in X)(x \rightarrow x=$ indeterminate or $x \rightarrow x \neq 1)$;

(NCI2) $(\exists x \in X)(\mathbf{1} \rightarrow \boldsymbol{x}=\boldsymbol{x})$ and $(\exists \boldsymbol{x} \in \boldsymbol{X})(\mathbf{1} \rightarrow \boldsymbol{x}=$ indeterminate or $1 \rightarrow \boldsymbol{x} \neq \boldsymbol{x})$; 
(NCI3) $(\exists x, y, z \in X$, with $x \neq y)(x \rightarrow(y \rightarrow z)=y \rightarrow(x \rightarrow z))$ and $(\exists x, y, z \in X$, with $x \neq y)(x \rightarrow(y \rightarrow z)=$ indeterminate or $x \rightarrow(y \rightarrow z) \neq y \rightarrow(x \rightarrow z))$.

\section{Definition 5.2. (Anti-sophications)}

The Anti-sophication of the Law (totally outer-defined)

(AL) $(\forall x, y \in X)(x \rightarrow y \notin X)$.

The Anti-sophication of the Axioms (totally false)

(ACI1) $(\forall x \in X)(x \rightarrow x \neq 1)$;

(ACI2) $(\forall x \in X)(1 \rightarrow x \neq x)$;

(ACI3) $(\forall x, y, z \in X$, with $x \neq y)(x \rightarrow(y \rightarrow z) \neq y \rightarrow(x \rightarrow z))$.

Definition 5.3. A Neutro-CI-algebra is an alternative of CI-algebra that has at least a (NL) or at least one (NCIt), $\boldsymbol{t} \in$ $\{\mathbf{1}, \mathbf{2}, \mathbf{3}\}$, with no anti-law and no anti-axiom.

Definition 5.4. An Anti-CI-algebra is an alternative of CI-algebra that has at least an (AL) or at least one (NCIt), $t \in$ $\{1,2,3\}$.

A Neutro-BE-algebra ([7]) is a Neutro-CI-algebra or has (NBE), where

(NBE) $(\exists x \in X)(x \rightarrow \mathbf{1}=\mathbf{1})$ and $(\exists x \in X)(x \rightarrow \mathbf{1}=$ indeterminate or $x \rightarrow \mathbf{1} \neq \mathbf{1})$.

An Anti-BE-algebra ([7]) is an Anti-CI-algebra or has (ABE), where

$(\mathrm{ABE})(\forall \boldsymbol{x} \in \boldsymbol{X})(\boldsymbol{x} \rightarrow \mathbf{1} \neq \mathbf{1})$.

Note that any proper CI-algebra may be a Neutro-BE-algebra or Anti-BE-algebra.

Proposition 5.5. Every NeutroSelf-distributive CI-algebra is a Neutro-CI-algebra.

Proposition 5.6. Every AntiSelf-distributive CI-algebra is an Anti-BE-algebra.

Proposition 5.7. Every AntiCommutative CI-algebra is an Anti-BE-algebra.

\section{Conclusions}

In this paper, Neutro-CI-algebras and Anti-CI-algebras are introduced and discussed based on the definition of CI-algebras. By some examples we showed that these notions were different. Some of their properties were provided. We proved that any proper CI-algebra is a Neutro-BE-algebra or Anti-BE-algebra. Further, for every classical CIalgebra, it was shown that, if it is AntiSelf-distributive or AntiCommutative, then it is an Anti-BE-algebra.

Acknowledgment: The authors would like to thank the reviewers for their reading of the manuscript and their many insightful comments and suggestions.

Funding: This research received no external funding. 
Conflicts of Interest: The authors declare no conflict of interest.

\section{References}

[1] H.S. Kim, Y.H. Kim, On BE-algebras, Sci. Math. Jpn. vol. 66, no. 1, pp. 113-117, 2007.

[2] B.L. Meng, CI-algebras, Sci. Math. Jpn. vol. 71, no. 1, pp. 11-17, 2010.

[3] B.L. Meng, Atoms in CI-algebras and singular CI-algebras, Sci. Math. Jpn. vol. 72, no. 1, pp. 319-324, 2010.

[4] B. Piekart, A. Walendziak, On filters and upper sets in CI-algebras, Algebra and Discrete Mathematics, vol. 11, no. 1, pp. 109-115, 2011.

[5] A. Rezaei, A. Borumand Saeid, Commutative ideals in BE-algebras, Kyungpook Math. J. vol. 52, pp. 483-494, 2012. Doi:10.5666/KMJ.2012.52.4.483.

[6] A. Rezaei, A. Borumand Saeid, R.A. Borzooei, Relation between Hilbert algebras and BE-algebras, Applications and Applied Mathematics, vol. 8, no. 2, pp. 573-584, 2013.

[7] A. Rezaei, F. Smarandache, On Neutro-BE-algebras and Anti-BE-algebras, International Journal of Neutrosophic Science, Volume 4 , Issue 1, pp. 08-15, 2020

[8] F. Smarandache, NeutroAlgebra is a Generalization of Partial Algebra, International Journal of Neutrosophic Science (IJNS), vol. 2, no. 1, pp. 8-17, 2020.

[9] F. Smarandache, Introduction to NeutroAlgebraic Structures and AntiAlgebraic Structures (revisited), Neutrosophic Sets and Systems, vol. 31, pp. 1-16, 2020. DOI: 10.5281/zenodo.3638232.

[10] F. Smarandache, Introduction to NeutroAlgebraic Structures and AntiAlgebraic Structures, in Advances of Standard and Nonstandard Neutrosophic Theories, Pons Publishing House Brussels, Belgium, Ch. 6, pp. 240-265, 2019. http://fs.unm.edu/AdvancesOfStandardAndNonstandard.pdf.

[11] A. Walendziak, On commutative BE-algebras, Sci. Math. Jpn. vol. 69, pp. 585-588, 2008.

[12] X. Zhang, X. Wu, F. Smarandache, M. Hu, Left (Right)-Quasi Neutrosophic Triplet Loops (Groups) and Generalized BE-algebras, Symmetry, 2018,10, 241. DOI: 10.3390/sym10070241. 